\title{
Geometric and Photometric Registration for Real-time Augmented Reality
}

\author{
Masayuki Kanbara and Naokazu Yokoya \\ Graduate School of Information Science, Nara Institute of Science and Technology \\ 8916-5 Takayama-cho, Ikoma-shi, Nara 630-0101, JAPAN \\ \{kanbara, yokoya \} @is.aist-nara.ac.jp
}

\begin{abstract}
This paper proposes an augmented reality system with correct representation of shading and shadow. To realize a seamless augmented reality system, we need to resolve some problems. Especially, the geometric and photometric registration problems are important. These problems require the position of light sources and user's viewpoint. The proposed system resolves the problems using a 3D marker which combines a $2 D$ square marker and a mirror ball. The $2 D$ marker and the ball are used to estimate the relationship between the real and virtual worlds and the positions of light sources in the real world, respectively.
\end{abstract}

\section{Introduction}

Augmented reality produces an environment in which virtual objects are superimposed seamlessly on user's view of the real environment. Augmented reality has received a great deal of attention as a new method for displaying information or increasing the reality of virtual environments [1]. To implement an augmented reality system, we must solve some problems. When virtual objects should be superimposed on the place as if they really exist in the real world, the geometric and photometric registration problems are especially important. The photometric registration problem requires the consistency between light sources in the virtual world and those in the real world.

One of the major approaches to the geometric registration between the real and virtual worlds is a vision-based method. The methods, which are sometimes referred to as vision-based tracking or registration, estimate the position and orientation of user's viewpoint from images captured by a camera attached at the user's viewpoint. Some methods use square markers[2].

On the other hand, to resolve the photometric problem, the positions of light sources in the real world should be estimated in real time. One of the methods acquires a lighting environment by photographing a mirror ball[3]. The other method estimates the illumination distribution in the real world using cast shadow regions[4]. However, this method cannot estimate the lighting environment in real time.

This paper describes an augmented reality system which resolves both the geometric and photometric registration problems using a $3 \mathrm{D}$ marker consisting of a $2 \mathrm{D}$ square marker and a mirror ball, which is shown in Figure 1. We estimate a simple light source map observing a mirror ball.

\section{Geometric and Photometric Registration}

\subsection{Outline of the proposed method}

Figure 2 illustrates the outline of the proposed method. First, a camera captures a 3D marker which is composed of a 2D square marker and a mirror ball and is placed in the real scene. The relationship between the 2D marker and the mirror ball and their size are known. Second, the relationship between the marker and camera coordinate system is estimated by detecting the region of the $2 \mathrm{D}$ square marker using an existing method[2]. Using the result, the mirror ball in the image is detected. Next, a simple light source map which represents a distribution of light sources in the real scene is estimated from the mirror ball image. Finally, a virtual object is rendered by using the estimated light source map.

The rest of the section describes the geometric registration and the estimation of light source map in some more detail.

\subsection{Geometric registration}

In the geometric registration process, a 2D square region is detected based on the size, color and shape of the maker. The model-view matrix, which represents the relationship between the marker and the camera, is estimated by identifying the corners of the markers[2].

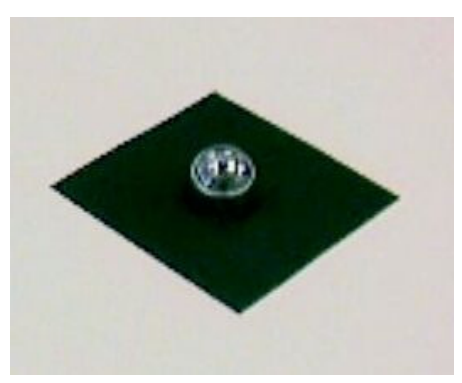

Figure 1. A 3D Marker 


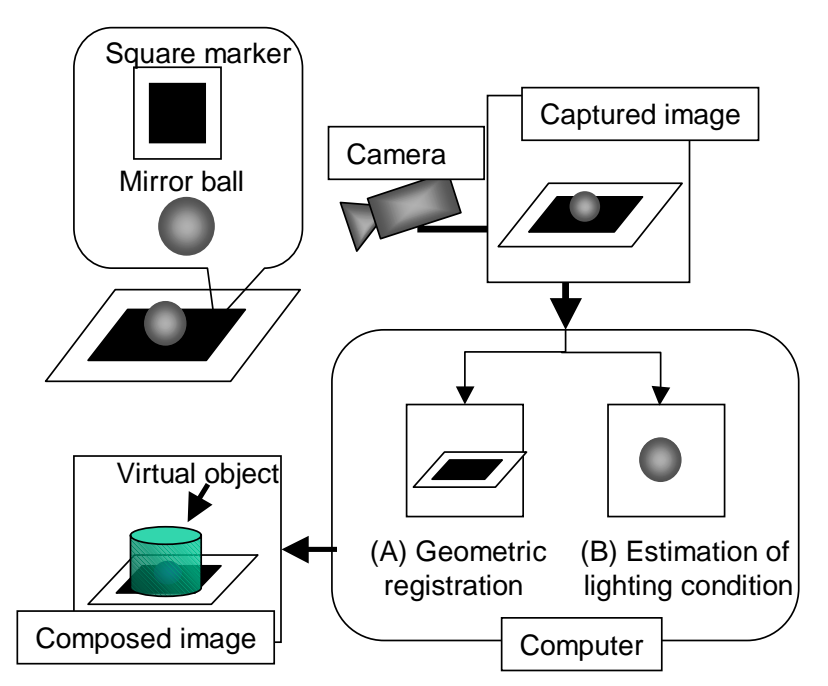

Figure 2. Flow diagram of the method

\subsection{Estimation of lighting environment}

The region of mirror ball in the captured image can be easily determined by using the model-view matrix estimated in Section 2.1 and the relationship between the 2D square marker and the mirror ball. The brightness of a light source in the real world is determined by intensities of pixels in the region of the mirror ball. Next, the directions of light sources are estimated by using the camera pose and surface normals of mirror ball points corresponding to the detected pixels.

\section{Experiment}

In the prototype system, an IEEE1394 camera (SONY: DFW-VL500) captures a 3D marker placed in the real world. The captured image is fed into a desktop PC (CPU P4 2GHz, Memory 256MB), and a composed image is generated.

Figure 3 shows experimental results. Figure 3(a) illustrates a region of mirror ball in the captured images, the brightness of light source is estimated from the intensity of pixels in the region. In the experiment, the size of the mirror ball region is about $20 \times 20$ pixels. Figure 3 (b) shows an estimated light source map in two different views. The size of sphere represents the brightness of each light source. Figure 3(c) shows a composed image in which a virtual object (teapot) is merged into the real world with geometric and photometric consistency. In the experiment, the frame rate is 15 frames per second.

\section{Conclusion}

This paper have described an augmented reality system which resolves both the geometric and photometric registration problems using a 3D marker composed of a 2D square marker and a mirror ball. The proposed method is characterized by the estimation of a light source map in real time.
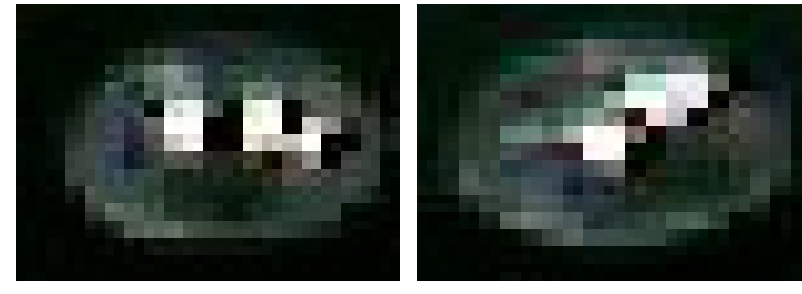

(a) Region of mirror ball in two views

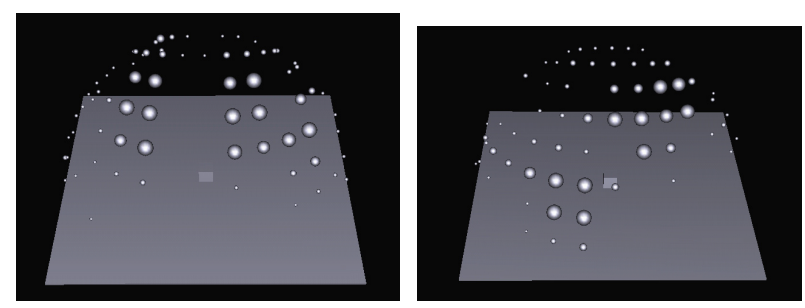

(b) Light source map in two views

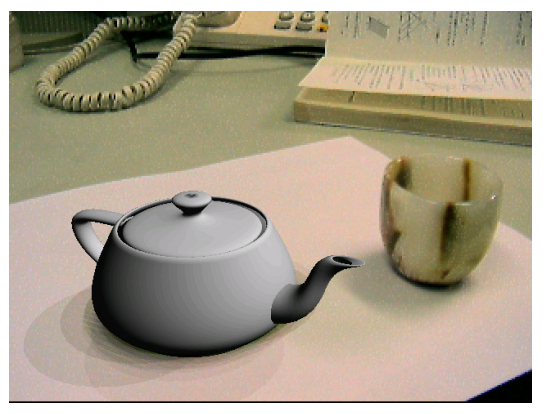

(c) Composed image

Figure 3. Experimental results

Acknowledgment This research is partially supported by Core Research for Evolutional Science and Technology (CREST) Program "Advanced Media Technology for Everyday Living" of Japan Science and Technology Corporation(JST) and also by the Grant-in-Aid for Scientific Research from the Ministry of Education, Culture, Sports, Science and Technology.

\section{References}

[1] M. Kanbara, T. Okuma, H. Takemura and N. Yokoya: "A Stereoscopic Video See-through Augmented Reality System Based on Real-time Vision-based Registration," Proc. IEEE Virtual Reality 2000, pp. 255-262, 2000.

[2] H. Kato, M. Billinghurst, I. Poupyrev, K. Imamoto and K. Tachibana: "Virtual Object Manipulation on a Table-top AR Environment," Proc. IEEE/ACM Int. Sympo. on Augmented Reality, pp. 111-119, 2000.

[3] P. Debevec: "Rendering Synthetic Objects into Real Scenes: Bridging Traditional and Image-based Graphics with Global Illumination and High Dynamic Range Photography," Proc. SIGGRAPH'98, pp. 189-198, 1998.

[4] I. Sato, Y. Sato and K. Ikeuchi: "Illumination Distribution from Brightness in Shadows: Adaptive Estimation of Illumination Distribution with Unknown Reflectance Properties in Shadow Regions," Proc. ICCV’99, pp. 875-882, 1999. 